\title{
Born-Infeld Corrections to the Entropy Function of Heterotic Black Holes
}

\author{
B. Chandrasekhar* \\ Institute for Studies in Theoretical Physica and Mathematics (IPM), P.O. Box 19395-5531, Tehran, India
}

Received on 17 November, 2006

\begin{abstract}
We use the black hole entropy function to study the effect of Born-Infeld terms on the entropy of extremal black holes in heterotic string theory in four dimensions. We find that after adding a set of higher curvature terms to the effective action, attractor mechanism works and Born-Infeld terms contribute to the stretching of near horizon geometry. In the $\alpha^{\prime} \rightarrow 0$ limit, the solutions of attractor equations for moduli fields and the resulting entropy, are in conformity with the ones for standard two charge black holes.
\end{abstract}

Keywords: Extremal black holes; String theory; Black hole entropy. Born-Infeld terms

\section{INTRODUCTION}

String theories in their low-energy limit give rise to effective models of gravity in higher dimensions which involve an infinite series of higher curvature terms. The higher curvature corrections to the effective action considered of interest come from two sources, to wit, $\alpha^{\prime}$ corrections and string loop corrections in a given string theory. There has been considerable work on understanding the role of these higher curvature terms from various points of view, especially with regard to black hole physics [1]-[35]. One of the contexts which has received considerable attention, is the effect of higher curvature terms on the Black Hole entropy [9]-[12]. Intriguingly, the inclusion of $\alpha^{\prime}$ corrections in the low energy effective action leads to a resolution of naked singularities for certain classical solutions in string theory [13] and then the solutions describe black holes with a regular horizon. In fact, the effect of these higher derivative corrections for the near horizon geometry were anticipated from the 'stretched horizon' proposal in string theory [14]-[18]. As a consequence of these corrections, the black hole entropy also comes out to be finite.

In the presence of higher curvature terms, there are deviations from Bekenstein-Hawking area law. One of the hallmarks of string theory is the exact matching of black hole entropy computed from microscopic counting of the degeneracy of string states [19-21] and the macroscopic calculation from the solution carrying the same charges, even in the presence of higher curvature interactions [20]-[35]. Even more so, in some cases, the sub leading corrections to the entropy of certain black holes, which already have non-zero horizon area classically, have also been computed. These computations further the proposals relating extremal black holes and elementary string states [14, 23, 36].

An important ingredient of the black hole entropy computations involving higher derivative terms, is the calculational tool provided by supersymmetry. In particular, a rich structure has emerged out of the study of BPS black holes in $\mathcal{N}=2$ supersymmetric string theories. One of the remarkable features of black holes in these theories is the attractor mechanism,

* Present address: Instituto de Física Teórica, Universidade Estadual Paulista (UNESP), Rua Pamplona 145, Bela Vista, 01405-900, São Paulo, SP, Brazil. whereby, the scalar fields are drawn to fixed points as they approach the horizon [37]-[39]. In other words, the near horizon values of the scalar fields are independent of the asymptotic values of these moduli fields and they get fixed in terms of the charges carried by the black hole. As a result, the black hole solution and the associated entropy near the horizon is determined completely in terms of the conserved charges akin to the black hole. The entropy of these black holes has been calculated both in the supergravity approximation and even after the inclusion a certain set of higher curvature terms in the generalized prepotential. The result agrees with the microscopic counting done on the brane system they describe.

More recently, a generalized attractor mechanism has been formulated without making explicit use of supersymmetry and in the presence of higher derivative terms(see [33] for discussion with supersymmetry). There have been various approaches ${ }^{1}$ to this subject [28, 41]-[48]. In particular, an 'entropy function' formalism was put forward in [42] to study the role of higher derivative terms on the entropy of extremal black holes from the near horizon geometry, using Wald's entropy formula. Also, a c-extremization procedure was proposed in [43] assuming a near horizon geometry which contains an $\mathrm{AdS}_{3}$ factor.

In the entropy function formalism, to a first order approximation, it is assumed that the structure of near horizon geometry of the extremal black holes in this theory remains $A d S_{2} \times S^{2}$ in four dimensions, even after the inclusion of higher derivative terms in the action $[16,33]$. Based on this assumption, the black hole entropy is then understood as the Legendre transform of Lagrangian density $[42,49]$ multiplied by $2 \pi$, confirming the proposal of [41]. Using the above approach, the entropy of two, four and eight charge black holes in heterotic string theory was calculated in [50]. Intriguingly, it is possible to perform the calculation in a regime, where all the higher derivative terms come purely from $\alpha^{\prime}$ corrections. In this regime, the string loop corrections can be neglected. Therefore in [50], the corrections to black hole entropy occurring from the inclusion of specific class of higher deriva-

\footnotetext{
${ }^{1}$ For earlier work where attractor mechanism was understood on the basis of regularity of the geometry and various moduli fields near the horizon see [40].
} 
tive terms, namely, Gauss-Bonnet terms was computed. The Gauss-Bonnet terms appear at order $\alpha^{\prime}$ in the heterotic string effective action [51,52]. The extremization of 'entropy function' with respect to the moduli, then determines the generalized attractor equations. Attractor equations fix the values of moduli in terms of the charges carried by the black hole. The results can be generalized to include higher Gauss-Bonnet densities, based on Lovelock type of actions [53]. All the same, it is not yet clear why only a particular set of higher curvature corrections should be added to a given system. A possible hint is the anomaly approach to black hole entropy [43]. For some of the recent developments, see [54]-[62].

It is to be noted that in the aforementioned works, only the higher curvature corrections ensuing from the gravity side were included and their effect on the entropy was studied. However, if one is to consider the Maxwell fields coupled to a gravitational action, which also includes string generated corrections at higher orders in $\alpha^{\prime}$, then, it is naturally important to consider string generated corrections to the electromagnetic field action as well. Such corrections come from a coupling of abelian gauge fields to open bosonic or open superstrings. To be precise, it is known that, just like the Gauss-Bonnet terms, there are Born-Infeld terms [63], which appear as higher order $\alpha^{\prime}$ corrections to the Maxwell action [64]-[73].

However, explicit corrections of Born-Infeld type terms on the entropy of extremal black holes have not been computed thus far and their exact dependence is not known. Furthermore, it is important to study attractor mechanism in more general situations where there are higher order gauge and gravitational terms in the effective action. It is to be noted that the effect of these terms on the entropy of black holes is in general hard to compute analytically. It is the purpose of this letter to consider the effect of a certain class of string generated corrections to the electromagnetic part, appearing in the low energy limit of heterotic string theory and study their effect on the 'entropy function' and attractor mechanism. In particular, we shall modify the Maxwell part of the heterotic string action by including a more general Born-Infeld part minimally coupled to the dilaton and other scalar fields. In fact, the derivative independent part of the Born-Infeld action is exact to all orders in $\alpha^{\prime}$. Considering the analogy between the Gauss-Bonnet and the Born-Infeld terms, which are on similar footing with regard to string corrections on the gravity side and Maxwell side, respectively, it is important to include both these corrections simultaneously.

On more important grounds, since the inclusion of higher curvature terms gives rise to a finite area of the horizon of small black holes in heterotic string theory, it is interesting to explore whether the Born-Infeld terms have any role to play in this regard. Analogs of Reissner-Nordstrom Black hole solutions in Born-Infeld theories coupled to gravity and with general Lovelock type actions and dilaton, are being studied in literature [74]-[77]. However, while working with a complete solution, studying the attractor mechanism requires a different methodology, especially in the presence of higher curvature terms in the action; for instance, see [44, 78]. The entropy function formalism put forward in [42], allows us to compute the entropy of an extremal configuration from the near hori- zon geometry, without the explicit knowledge of a complete solution. Thus in this work, our approach would be to study the attractor mechanism and entropy of such black holes in the near horizon limit, by directly adding the relevant terms to the action, in the same vein as [50]. A complete study of attractor mechanism with the full solution, we leave for a future work. The near horizon analysis in fact already shows some interesting features, as we will see. Notice that we are considering an extremal configuration, where it can be assumed that the solution near the horizon does not get corrected by adding $\alpha^{\prime}$ corrections to the effective action. Of course, it is not assured that this will be the case, if a similar analysis is to be repeated for non-extremal black holes [73].

It is useful to recollect that the importance of Born-Infeld terms in the study of extremal black holes has been emphasized by Gibbons and Rasheed in [79]. It was noted that virtual black holes going around closed loops may induce corrections to the Maxwell lagrangian, which, at the string tree level, may be of the Born-Infeld type, though there are other theories giving non-linear electrodynamics in four dimensions. Extreme electrically charged black holes in Born-Infeld theories exist and the 'double degenerate' horizon occurs at $r_{H}=\sqrt{\frac{e^{2}}{2 \pi}-\frac{1}{32 \pi b^{2}}}$, where $e$ is the electric charge. Thus, there appears a natural bound on the electric charge for the existence of consistent solutions of the Reissner-Nordstrom type, albeit, with negative binding energy [2, 79](see also [80]). It is further interesting to address what these extremal configurations correspond to in terms of elementary string states. A possible hint is that, the charge to mass ratio of the BornInfeld black holes closely matches the result for the Maxwell Reissner-Nordstrom black holes [79]. In this paper, we attempt to compute the entropy of such extremal configurations in heterotic string theory with Born-Infeld corrections.

The rest of the paper is organized as follows. In section II, we write down the low-energy effective action of heterotic string theory in four dimensions with Born-Infeld type corrections and determine the 'entropy function' for a four charge black hole. In section III, we study the attractor mechanism in two charge black holes in this theory and show that the solutions of attractor equations are consistent only after the addition of higher curvature terms on the gravity side. We also illustrate that our results are in conformity with the standard results for two charge black holes in heterotic string theory, in the $\alpha^{\prime} \rightarrow 0$ limit. Our conclusions are summarized in section IV.

\section{ENTROPY FUNCTION WITH BORN-INFELD CORRECTIONS}

Our starting point is the low-energy effective action of heterotic string theory on $\mathcal{M} \times S^{1} \times \widetilde{S}^{1}$, where $\mathcal{M}$ is some four manifold. We follow the notations and conventions of [50] and review certain aspects needed for our purposes. Thus, we work in the supergravity approximation, where the four dimensional fields relevant for studying the black hole solution are related to the ten dimensional string metric $G_{M N}^{(10)}$, 
anti-symmetric tensor field $B_{M N}^{(10)}$ and the dilaton $\Phi^{(10)}$ as fol- $\quad$ lows [50, 81]:

$$
\begin{aligned}
& \Phi=\Phi^{(10)}-\frac{1}{4} \ln \left(G_{99}^{(10)}\right)-\frac{1}{4} \ln \left(G_{88}^{(10)}\right)-\frac{1}{2} \ln V_{\mathcal{M}}, \\
& S=e^{-2 \Phi}, \quad R=\sqrt{G_{99}^{(10)}}, \quad \widetilde{R}=\sqrt{G_{88}^{(10)}}, \\
& G_{\mu \nu}=G_{\mu \nu}^{(10)}-\left(G_{99}^{(10)}\right)^{-1} G_{9 \mu}^{(10)} G_{9 v}^{(10)}-\left(G_{88}^{(10)}\right)^{-1} G_{8 \mu}^{(10)} G_{8 v}^{(10)} \\
& A_{\mu}^{(1)}=\frac{1}{2}\left(G_{99}^{(10)}\right)^{-1} G_{9 \mu}^{(10)}, \quad A_{\mu}^{(2)}=\frac{1}{2}\left(G_{88}^{(10)}\right)^{-1} G_{8 \mu}^{(10)}, \\
& A_{\mu}^{(3)}=\frac{1}{2} B_{9 \mu}^{(10)}, \quad A_{\mu}^{(4)}=\frac{1}{2} B_{8 \mu}^{(10)} .
\end{aligned}
$$

Here, $V_{\mathcal{M}}$ is the volume of $\mathcal{M}$ measured in the string metric. The effective action governing the dynamics of these fields is:

$$
\begin{aligned}
& S=\frac{1}{32 \pi} \int d^{4} x \sqrt{-\operatorname{det} G} S\left[R_{G}+S^{-2} G^{\mu v} \partial_{\mu} S \partial_{v} S-R^{-2} G^{\mu v} \partial_{\mu} R \partial_{v} R-\widetilde{R}^{-2} G^{\mu v} \partial_{\mu} \widetilde{R} \partial_{v} \widetilde{R}\right. \\
& -R^{2} G^{\mu v} G^{\mu^{\prime} v^{\prime}} F_{\mu \mu^{\prime}}^{(1)} F_{v v^{\prime}}^{(1)}-\widetilde{R}^{2} G^{\mu v} G^{\mu^{\prime} v^{\prime}} F_{\mu \mu^{\prime}}^{(2)} F_{v v^{\prime}}^{(2)}-R^{-2} G^{\mu v} G^{\mu^{\prime} v^{\prime}} F_{\mu \mu^{\prime}}^{(3)} F_{v v^{\prime}}^{(3)} \\
& \left.-\widetilde{R}^{-2} G^{\mu v} G^{\mu^{\prime} v^{\prime}} F_{\mu \mu^{\prime}}^{(4)} F_{v v^{\prime}}^{(4)}\right] \quad+\text { higher derivative terms + string loop corrections. }
\end{aligned}
$$

Here, $A_{\mu}^{(1)}$ and $A_{\mu}^{(3)}$ couple to the momentum and winding numbers along the $x^{9}$ direction, whereas the fields $A_{\mu}^{(2)}$ and $A_{\mu}^{(4)}$ couple to the momentum and winding numbers along the $x^{8}$ direction with the following identifications [50]:

$$
q_{1}=\frac{1}{2} n, \quad q_{3}=\frac{1}{2} w, \quad p_{2}=4 \pi \widetilde{N}, \quad p_{4}=4 \pi \widetilde{W} .
$$

where $q_{1}$ and $q_{3}$ are electric charges and $p_{2}$ and $p_{4}$ are magnetic charges. In [16], it was shown that the black hole solution in the limit where the $\alpha^{\prime}$ corrections are important, is completely universal and higher derivative terms give corrections in a specific way. In this work, we shall consider the Born-Infeld terms and study the entropy function in the resulting theory.

The Lagrangian density describing Born-Infeld theory in arbitrary space-time dimensions is:

$$
\mathcal{L}_{F}=4 b\left\{\sqrt{\mid \operatorname{det} G}-\sqrt{\left|\operatorname{det}\left(G_{\mu \nu}+\frac{1}{\sqrt{b}} F_{\mu \nu}\right)\right|}\right\},
$$

Restricting to four space-time dimensions and using the Monge gauge (or static gauge) the determinant in the above action can be expanded to obtain:

$$
\mathcal{L}_{B I}=4 b S^{-1}\left\{1-\left[1+\frac{S^{2}}{2 b} F^{2}-\frac{S^{4}}{16 b^{2}}(F \star F)^{2}\right]^{\frac{1}{2}}\right\}
$$

where we have also included the dilatonic coupling. Here $\star F$ is dual to the Maxwell tensor which does not contribute in the following discussion. In the context of string theory, the parameter $b$ is related to string tension $\alpha^{\prime}$ as: $b^{-1}=\left(2 \pi \alpha^{\prime}\right)^{2}$. Note that in the $b \rightarrow \infty$ limit the action reduces to the Maxwell system. We continue to retain the Born-Infeld parameter, so as to make a comparison with [50]. We set $\sqrt{\alpha^{\prime}}=4$ and the Newton's constant $G_{N}=2$. With these conventions, $b^{-1}=$ $(32 \pi)^{2}$.

Let us then take the effective field theory action of heterotic string in the supergravity limit to be:

$$
\begin{aligned}
& \mathcal{S}=\frac{1}{32 \pi} \int d^{4} x \sqrt{-\operatorname{det} G} S\left[R_{G}+S^{-2} G^{\mu v} \partial_{\mu} S \partial_{v} S \quad-R^{-2} G^{\mu v} \partial_{\mu} R \partial_{v} R\right. \\
& \left.-\widetilde{R}^{-2} G^{\mu \nu} \partial_{\mu} \widetilde{R} \partial_{v} \widetilde{R}-R^{2} \mathcal{L}_{B I}^{(1)}-\widetilde{R}^{2} \mathcal{L}_{B I}^{(2)}-R^{-2} \mathcal{L}_{B I}^{(3)}-\widetilde{R}^{-2} \mathcal{L}_{B I}^{(3)}\right]
\end{aligned}
$$

and here the higher derivative terms and string loop corrections denote the ones coming from gravity side. The Born-
Infeld terms can be written in a useful form as:

$$
\mathcal{L}_{B I}^{i}=4 b S^{-2}\left\{1-\left[1+\frac{S^{2}}{2 b} G^{\mu v} G^{\mu^{\prime} v^{\prime}} F_{\mu \mu^{\prime}}^{(i)} F_{v v^{\prime}}^{(i)}\right]^{\frac{1}{2}}\right\} \text {. }
$$


We have adjusted the dilaton couplings and the coefficients in such a way that in the $\alpha^{\prime} \rightarrow 0$ limit, we recover the action given in eqn. (2.2). The particular coupling of dilaton appearing in the Born-Infeld terms can also be motivated from $S$-duality arguments from Type-I superstring theory.

Let us now consider an extremal charged black hole solution in this theory, in its near horizon limit, with $A d S_{2} \times S^{2}$ as the near horizon geometry. Let us further assume that the string generated corrections, do not spoil this property, and only give corrections to various parameters characterizing the near horizon geometry, thereby, changing relations between them. Thus, with all these conventions for the Born-Infeld corrections in the effective action (2.6), let us consider the near horizon geometry to be [50]:

$$
\begin{array}{r}
d s^{2}=v_{1}\left(-r^{2} d t^{2}+\frac{d r^{2}}{r^{2}}\right)+v_{2}\left(d \theta^{2}+\sin ^{2} \theta d \phi^{2}\right), \\
S=u_{S}, \quad R=u_{R}, \quad \widetilde{R}=u_{\widetilde{R}}, \\
F_{r t}^{(1)}=e_{1}, \quad F_{r t}^{(3)}=e_{3}, \quad F_{\theta \phi}^{(2)}=\frac{p_{2}}{4 \pi}, \quad F_{\theta \phi}^{(4)}=\frac{p_{4}}{4 \pi},
\end{array}
$$

where $v_{1}, v_{2}$ are the radii of $A d S_{2}$ and $S^{2}, e_{1}, e_{3}$ are radial electric fields and $p_{2}, p_{4}$ are the radial magnetic fields, $u_{S}$ stands for the dilaton and $u_{R}, \widetilde{u}_{R}$ characterize the radii of $S^{1}$ and $\widetilde{S}^{1}$. All these objects are constants in the near horizon limit.

Following the prescription in [50], let us consider the function:

$$
f\left(u_{S}, u_{R}, \widetilde{u}_{R}, v_{1}, v_{2}, e_{1}, e_{3}, p_{2}, p_{4}\right) \equiv \int d \theta d \phi \sqrt{-\operatorname{det} G} \mathcal{L} .
$$

The equations of motion of scalar fields and the metric, equivalent to Einstein's equations, can be obtained by extremizing the function $f$ with respect to $u_{S}, u_{R}, \widetilde{u}_{R}, v_{1}$ and $v_{2}$. The gauge field equations are derived as follows. It is usual to define the tensor $G^{\mu v}$ as:

$$
G^{(1) \mu \nu}=-\frac{1}{2} \frac{\partial \mathcal{L}_{F}^{(1)}}{\partial F_{\mu \nu}^{(1)}}=-\frac{S^{2} R^{2} F^{(1) \mu \nu}}{\sqrt{1+\frac{S^{2}}{2 b} F^{(1) 2}}}
$$

so that $G^{(1) \mu v} \approx F^{(1) \mu v}$ for weak fields (and with similar results for other gauge fields). The tensor $G^{\mu \nu}$ satisfies the electromagnetic field equations, the non-trivial components of which are:

$$
\partial_{r}\left(\sqrt{-\operatorname{det} G} G^{(i) r t}\right)=0, \quad \partial_{r} G^{(i) \theta \phi}=0 .
$$

where $i$ runs over all the gauge fields. As in [50], even for the present case, these gauge field equations are satisfied for the background (2.8). The entropy associated with this black hole can be calculated using the formula [9-12]:

$$
S_{B H}=8 \pi \frac{\partial \mathcal{L}}{\partial R_{r t r t}} g_{r r} g_{t t} A_{H}
$$

where $A_{H}$ denotes the area of the event horizon. This formula can be brought in to a form, where the black hole entropy can be obtained from the Legendre transform of $f$ as [42]:

$$
S_{B H}=2 \pi\left(e_{i} \frac{\partial f}{\partial e_{i}}-f\right),
$$

where the function $f$ is defined in eqn. (2.9). In our case, for the action given in eqn. (2.6), $f$ turns out to have the form:

$$
\begin{aligned}
& f\left(u_{S}, u_{R}, \widetilde{u}_{R}, v_{1}, v_{2}, e_{1}, e_{2}, p_{2}, p_{4}\right) \\
& =\frac{1}{8} v_{1} v_{2} u_{S}\left[-\frac{2}{v_{1}}+\frac{2}{v_{2}}+4 b u_{R}^{2} u_{S}^{-2}\left\{1-\left[1-\frac{u_{S}^{2}}{b} \frac{e_{1}^{2}}{v_{1}^{2}}\right]^{\frac{1}{2}}\right\}\right. \\
& +4 b u_{R}^{-2} u_{S}^{-2}\left\{1-\left[1-\frac{u_{S}^{2}}{b} \frac{e_{3}^{2}}{v_{1}^{2}}\right]^{\frac{1}{2}}\right\}+4 b u_{\widetilde{R}}^{2} u_{S}^{-2}\left\{1-\left[1+\frac{u_{S}^{2}}{b} \frac{p_{2}^{2}}{16 \pi^{2} v_{2}^{2}}\right]^{\frac{1}{2}}\right\} \\
& \left.+4 b u_{\widetilde{R}}^{-2} u_{S}^{-2}\left\{1-\left[1+\frac{u_{S}^{2}}{b} \frac{p_{4}^{2}}{16 \pi^{2} v_{2}^{2}}\right]^{\frac{1}{2}}\right\}\right] .
\end{aligned}
$$

Now, we would like to take a Legendre transform of the above function and calculate the entropy function defined as [42, 50]:

$$
F(\vec{u}, \vec{v}, \vec{e}, \vec{q}, \vec{p}) \equiv 2 \pi\left(e_{i} q_{i}-f(\vec{u}, \vec{v}, \vec{e}, \vec{p})\right),
$$

where,

$$
q_{i} \equiv \frac{\partial f}{\partial e_{i}}
$$

For the function $f$ given in eqn. (2.14), we get:

$$
q_{1,3}=e_{1,3} \frac{v_{2} u_{S} u_{R}^{2}}{2 \sqrt{b v_{1}^{2}-e_{1,3}^{2} u_{S}^{2}}} .
$$


Using the above, the entropy function computed from eqn. (2.15) takes the form:

$$
\begin{aligned}
& F\left(u_{S}, u_{R}, \widetilde{u}_{R}, v_{1}, v_{2}, q_{1}, q_{3}, p_{2}, p_{4}\right)=\frac{\pi}{2}\left(v_{2}-v_{1}\right) u_{S} \\
& +\pi \sqrt{b} \frac{v_{1}}{u_{S}}\left[\sqrt{4 q_{1}^{2}+b v_{2}^{2} u_{R}^{4}}-\sqrt{b} v_{2} u_{R}^{2}+\sqrt{4 q_{3}^{2}+b v_{2}^{2} u_{R}^{-4}}-\sqrt{b} v_{2} u_{R}^{-2}\right] \\
& +\sqrt{b} \frac{v_{1}}{4 u_{S}}\left[\sqrt{u_{S}^{2} p_{2}^{2}+16 \pi^{2} b v_{2}^{2}}-\pi \sqrt{b} v_{2} \widetilde{u}_{R}^{2}+\sqrt{u_{S}^{2} p_{4}^{2}+16 \pi^{2} b v_{2}^{2}}-\pi \sqrt{b} v_{2} \widetilde{u}_{R}^{-2}\right] .
\end{aligned}
$$

Now, the attractor equations can be derived from:

$$
\frac{\partial F}{\partial u_{s}}=0, \quad \frac{\partial F}{\partial v_{1}}=0, \quad \frac{\partial F}{\partial v_{2}}=0, \quad \frac{\partial F}{\partial u_{R}}=0, \quad \frac{\partial F}{\partial \widetilde{u}_{R}}=0 .
$$

In a generic case, the attractor equations may or may not be solvable. In fact, in the case of four charge black hole we started with, it is difficult to solve the attractor equations analytically. In particular, the attractor equations determining $u_{S}, v_{1}, v_{2}$ are complicated. However, the attractor equations for $u_{R}$ and $\widetilde{u}_{R}$ can still be written in terms of other moduli fields, in a suggestive manner. For convenience, we give the result for $u_{R}$ in terms of the radial electric fields $e_{1}$ and $e_{3}$ :

$$
u_{R}{ }^{4}=\frac{\left[\sqrt{b v_{1}^{2}-e_{3}^{2} u_{S}^{2}}-\sqrt{b} v_{1}\right]}{\left[\sqrt{b v_{1}^{2}-e_{1}^{2} u_{S}^{2}}-\sqrt{b} v_{1}\right]},
$$

and for $\widetilde{u}_{R}$, we have:

$$
\widetilde{u}_{R}^{4}=\frac{\left[\sqrt{16 \pi^{2} b v_{2}^{2}+p_{4}^{2} u_{S}^{2}}-4 \pi \sqrt{b} v_{2}\right]}{\left[\sqrt{16 \pi^{2} b v_{2}^{2}+p_{2}^{2} u_{S}^{2}}-4 \pi \sqrt{b} v_{2}\right]} .
$$

One can check that in the $b \rightarrow \infty$ limit, the standard result for $u_{R}$ and $\widetilde{u}_{R}$ is recovered [50] and these results will be quoted below. Notice that the expressions for the entropy function and final result for the moduli fields are explicitly duality invariant.

\section{TWO CHARGE BLACK HOLES}

As already mentioned, it is in general hard to solve the attractor equations in the case when all four charges are present. Hence, we first set the magnetic charges to zero. We shall comment on the dyonic case in the discussion section.

We are dealing with a small black hole, for which the area of the horizon and also the entropy vanishes to leading order [14]. Thus, in this case, the higher curvature corrections on the gravity side and even the Born-Infeld corrections on the Maxwell side are supposed to be important. It is known that upon the consideration of higher derivative corrections, a nonzero horizon emerges. In this case, the attractor equations of the moduli fields are modified and the solutions receive corrections. In this section, we shall analyze the corrections to solutions of various moduli fields and to the entropy from the Born-Infeld terms.
To start with, we do not include any higher curvature corrections on the gravity side. In other words, we check whether the Born-Infeld terms independently play any role near the horizon of a black hole. Therefore, we work with the BornInfeld corrected effective action given in eqn. (2.6). For convenience, we deal the case where $q_{1}=q_{3}=q$, keeping in mind that there are still two electric fields, but, with equal magnitudes of charges and $p_{2}=p_{4}=0$. That is, we only keep the charges associated with the momentum and winding numbers along the direction $x^{9}$ non-zero.

To this end, terms in the Entropy function in eqn. (2.18) that are relevant for the electrically charged case are:

$$
\begin{gathered}
F\left(u_{S}, v_{1}, v_{2}, q\right)= \\
\pi\left[\frac{u_{S}}{2}\left(v_{2}-v_{1}\right)+2 \sqrt{b} \frac{v_{1}}{u_{S}}\left(\sqrt{4 q^{2}+b v_{2}^{2}}-\sqrt{b} v_{2}\right)\right]
\end{gathered}
$$

Notice that we have already solved the attractor equation for the field $R$, which denotes the radius of the circle $S^{1}$, with the result $u_{R}=1$. This solution is better found before taking the Legendre transform, as it turns out to be a simpler equation when written in terms of electric fields, as in eqn. (2.20). Using the relations in eqn.(2.19), one can derive the attractor equations for various moduli fields from eqn. (??). Solving the attractor equations, one finds that (let us use $|q|$ to avoid negative signs in the square root):

$$
v_{2}=2 v_{1}=\frac{4|q|}{\sqrt{3 b}}, \quad u_{S}^{2}=-\left(\frac{64 b}{3}\right)^{\frac{1}{2}}|q| .
$$

It turns out that the solution for the dilaton squared with a positive sign is incompatible with rest of the attractor equations and the only solution is when the sign is negative (neglecting the trivial $q=0$ solution). Thus, the solutions of attractor equations are not consistent, which means that the Born-Infeld terms on their own are not enough to give a finite horizon and entropy to the black holes in this theory. It also signifies that black holes in this theory with only Born-Infeld terms do not have a nice extremal limit. This matches with the proposals in [77] about the non-existence of extremal limit for electrically charged black holes in Born-Infeld theories. The case when magnetic charges are also included remains to be checked.

In any case, let us appreciate the fact that solutions for the moduli fields $v_{1}$ and $v_{2}$, though inconsistent, turn out to be 
of the order of $\alpha^{\prime}$. Now, since there are $\alpha^{\prime}$ corrections coming from the gravity side, one might wonder whether adding these terms has any effect on the result (3.2). Especially, since we have set the magnetic charges to zero, comparing with the case in [50], it is probably fine to assume that the string loop corrections (inverse square root of $u_{S}$ ) are insignificant in this limit and $\alpha^{\prime}$ corrections are important.

Thus far, we have considered small black holes which have a vanishing horizon and entropy if only the leading order terms in the action are present. The addition of higher curvature terms changes this story. Thus, it is interesting to consider higher curvature terms which can give same order contribution to entropy as the Born-Infeld terms. With this in mind, we now include Gauss-Bonnet terms in the original action in eqn. (2.6) and look for solutions of attractor equations. Apriori, this may look strange since the Gauss-Bonnet term is an $\alpha^{\prime}$ correction, whereas the Born-Infeld term considered is exact and contains terms to all orders in $\alpha^{\prime}$. While, keeping only $\alpha^{\prime}$ corrections on both sides is reasonable, solving the attractor equations order by order in $\alpha^{\prime}$ is tedious. Thereupon, we resort to an exact treatment on the Born-Infeld side. Besides, the final result for entropy with Born-Infeld terms turns out to be of the order of $\alpha^{\prime}$, as seen from eqn. (3.2).

In [50], the corrections to the entropy from the GaussBonnet terms have already been computed and we use those results here. The terms added to the effective action of heterotic string theory were of the form :

$$
\Delta \mathcal{L}=C \frac{S}{16 \pi}\left\{R_{G \mu \nu \rho \sigma} R_{G}^{\mu \nu \rho \sigma}-4 R_{G \mu v} R_{G}^{\mu \nu}+R_{G}^{2}\right\},
$$

where $R_{G \mu v \rho \sigma}$ denotes the Riemann tensor computed using the string metric $G_{\mu v}$. The Gauss-Bonnet parameter is given as, $C=\alpha^{\prime} / 16$ and is equal to 1 according to the present notations. Due to the above term, solutions for various moduli fields get modified as (setting $p_{2}=p_{4}=0$ ) [50]:

$$
v_{1}=v_{2}=8, \quad u_{S}=\sqrt{q_{1} q_{3}} .
$$

In fact, the result for entropy gets corrected as:

$$
S_{B H}=8 \pi \sqrt{C q_{1} q_{3}},
$$

with the values of other moduli remaining unchanged.

In the present case, including the Gauss-Bonnet terms, the correction to the entropy function can be calculated with the following changes in the functions $f$ and $F$ :

$$
\Delta f=-2 u_{S}, \quad \Delta F=4 \pi u_{S},
$$

and the relation between $q_{i}$ and $e_{i}$ unchanged. Solving the attractor equations for $F+\Delta F$ following from eqn.(3.1) and (3.6), we have:

$$
\begin{aligned}
& v_{1}=C \frac{8}{3}\left(2+\sqrt{1-\frac{3 q^{2}}{16 b C^{2}}}\right), \\
& v_{2}=C \frac{8}{3}\left(1+2 \sqrt{1-\frac{3 q^{2}}{16 b C^{2}}}\right), \\
& u_{S}=C \sqrt{\frac{32 b}{3}}\left(1-\sqrt{1-\frac{3 q^{2}}{16 b C^{2}}}\right)^{\frac{1}{2}} .
\end{aligned}
$$

There are several things to note from the above solution. First of all, it is extremely interesting to note that now there are consistent solutions of the attractor equations, where the radii of $A d S_{2}, S^{2}$ and the dilaton, get fixed in terms of the charge $q$. This signifies the presence of attractor mechanism in this theory. Tracing back, one can see what happens to eqns. (3.7), if the Gauss-Bonnet term is set to zero. This leads us to a very important conclusion that the Gauss-Bonnet contribution provides a bound for the existence of extremal configurations and for the presence of attractor mechanism in this theory. This in turn brings about the importance of $\alpha^{\prime}$ corrections from the near horizon analysis. In fact, if the above analysis could be repeated in higher dimensions, with the higher GaussBonnet densities, then, there might be a suggested bound on the charges, from the coefficients of Lovelock terms to each order [53], putting a bound on the existence of extremal configurations.

Now as expected, the entire correction to the radius of $A d S_{2}$ and $S^{2}$ in eqn. (3.7) is of the order of $\alpha^{\prime}$. This is because the combination $b C^{2}=\frac{1}{4^{5} \pi^{2}}$, is dimensionless. Thus, to this order, various moduli fields receive corrections not just from the Gauss-Bonnet part [50], but also from the Born-Infeld part. Since these corrections to the horizon are of the same order, per se, the Born-Infeld corrections cannot be ignored. The aforementioned bound on the existence of extremal configurations in this theory is:

$$
q<8 \sqrt{3} \pi
$$

As long as this bound is satisfied, the solutions in eqn. (3.7) are consistent and attractor mechanism works.

One can draw further conclusions from the solutions for the moduli fields given in eqn. (3.7). One notices that the radii of $A d S_{2}$ and $S^{2}$ are not equal, unlike the correction given by a Gauss-Bonnet terms [50]. Such a correction to the radii from the Born-Infeld terms means that the near horizon geometry will no longer be conformally flat. This further signifies that the cosmological constant is also non-zero. Unbroken supersymmetry, if present would require that the radii of $A d S_{2}$ and $S^{2}$ should be equal. In fact, this is what is expected if one uses a supersymmetric attractor mechanism. Thus, the solutions in eqn. (3.7) should be non-supersymmetric, with the sign of $q^{2}$ chosen to be positive. This is consistent with the present formalism, where neither the solution nor the method of deriving the entropy function requires supersymmetry to be present. In the present example, taking $q^{2}$ to be negative is inconsistent and does not give any result in the corresponding non-BPS case.

Let us also note the fact that, if we take $\alpha^{\prime} \rightarrow 0$ limit in the solutions in eqn. (3.7), one clearly recovers the standard result, that $A d S_{2}$ and $S^{2}$ have the same radii, which is zero and the naked singularity reappears. Furthermore, the dilaton diverges, as is expected from the results in [50](since we did not include magnetic charges).

Let us now turn to the Born-Infeld corrections to the entropy of the extremal black holes in this theory. Using the solutions of the moduli fields in the entropy function gives us the entropy of the near horizon configuration in this theory. Without dwelling in to calculational details, let us present the 


$$
\begin{aligned}
S_{B H} & =4 \pi \frac{u_{S} v_{1}}{4}=\frac{32 \pi}{3} \sqrt{\frac{2}{3} \sqrt{b C^{3}}}\left(1-\sqrt{1-\frac{3 q^{2}}{16 b C^{2}}}\right)^{\frac{1}{2}}\left(2+\sqrt{1-\frac{3 q^{2}}{16 b C^{2}}}\right) \\
& =\frac{1}{12} \sqrt{\frac{2 \alpha^{\prime}}{3}}\left(1-\sqrt{1-(8 \sqrt{3} \pi)^{2} q^{2}}\right)^{\frac{1}{2}}\left(2+\sqrt{1-(8 \sqrt{3} \pi)^{2} q^{2}}\right)
\end{aligned}
$$

For an extremal black hole in the theory governed by the action in eqn. (2.6), with Gauss-Bonnet terms in addition, the entropy comes out to be as in eqn. (3.9). It is worth mentioning that the characteristic dependence of the Born-Infeld parameter on the entropy of extremal black holes has not been calculated before, either from the near horizon analysis or from the full solution. Although, the above results were derived in a rather simple set up, the importance increases, considering the fact that a similar calculation with all four charges turned on is already not possible analytically.

Let us look at the Born-Infeld contribution to the entropy of the extremal black holes in this theory. To ensure that our results are correct, it is important to check that taking appropriate limits in eqn. (3.9) and comparing it with the known results, we get the right answers. For instance, one can take the $b \rightarrow \infty$ limit which corresponds to recovering the results in the Maxwell regime considered in [50]. In this limit, keeping the Gauss-Bonnet parameter $C$ intact, for the entropy, one recovers the result $S_{B H}=8 \pi \sqrt{C q_{1} q_{3}}$, same as in eqn. (3.5). Furthermore, in eqn. (3.9), taking the $\alpha^{\prime} \rightarrow 0$ limit, one recovers the result for the entropy of small black holes, which vanishes due to the vanishing of horizon area. Moreover, in the results for the moduli fields in eqn. (3.7), one can check what happens if the charge $q$ is not present. In this case, one recovers the results of [50], where the correction to the horizon due to the Gauss-Bonnet terms is evident. Thus, our results for the moduli fields and the entropy have the correct limits and show the additional corrections due to the BornInfeld terms in the effective action. The above analysis also shows the importance of adding higher curvature terms for the attractor mechanism to work in this theory.

\section{DISCUSSION}

In this letter, we studied the effect of Born-Infeld on the entropy of small black holes in heterotic string theory in four dimensions and the attractor mechanism, using the entropy function formalism. We found that the near horizon geometry gets corrections from the Born-Infeld terms, which are of the same order as the Gauss-Bonnet contribution. The resulting entropy comes out to be finite and shows additional corrections due to the Born-Infeld terms. However, these corrections are inconsistent without the addition of Gauss-Bonnet terms. Moreover, the attractor mechanism works consistently only after the addition of such higher curvature terms to the effec- tive action. It is thus important to include all possible corrections to the horizon geometry at a given order in $\alpha^{\prime}$. In this work, we restricted ourselves to only two charge black holes in four dimensions. The case of a dyonic black hole is interesting, although the calculations involve solving coupled higher order algebraic equations. We have not been able to find any analytic solutions in this case. It is also important to include other types of terms in the calculation. For instance, it should be interesting to explore what happens when the S-duality invariant axionic coupling of the Born-Infeld and Gauss-Bonnet terms is considered in this theory.

It is to be noted that, in the entropy function formalism, it is implicit that all the scalar fields at the horizon take constant values. There exist other approaches to non-supersymmetric attractor mechanism, which make explicit use of the general solution and equations of motion [44]. In this picture, extremization of an effective potential, gives rise to the necessary attractor equations. In addition, there is sufficiency condition, which states that the extremum should also be a minimum. Furthermore, using a perturbative approach to study the corrections to the scalar fields and taking the backreaction corrections in to the metric, it is possible to show that the scalar fields are indeed drawn to their fixed values at the horizon. Here, the requirements are the existence of a double degenerate horizon solution, as in a Reissner-Nordstrom black hole.

Recently, the connection between the 'entropy function' and the effective potential approach has been detailed in [48] and non-supersymmetric attractors in $R^{2}$ gravities have been discussed in [78]. In the absence of supersymmetry, it cannot be guaranteed that the extremum of the effective potential will also be a minimum and hence, this must be checked on a case to case basis.

The analysis in this paper provides weightage to the existence of a consistent extremal limit for electrically charged black holes in Born-Infeld-Gauss-Bonnet theories. Motivated by the near horizon analysis in this work, it should be possible to show that there is no attractor mechanism for black holes in purely Einstein-Born-Infeld theories coupled to moduli fields. One might hope to see this feature in the non-existence of an extremum of the the effective potential of the theory. It is important to check this aspect for confirmation, using other methods of studying attractor mechanism. Finally, attractor mechanism seems to work only after the addition of other higher curvature terms from the gravity side, for example the Gauss-Bonnet term in this case. It is thus an extremely interesting and important problem to check these issues using 
the equations of motion explicitly for Einstein-Born-Infeld and Gauss-Bonnet systems coupled to moduli fields in four and higher dimensions, either by analytic or numerical methods [44, 78].

It is also important to remember that the formalism prescribed in [50] does not use any supersymmetry to derive attractor equations for the moduli fields and to calculate the entropy. Further, the Gauss-Bonnet term added to the action did not come with its supersymmetric partner. Similarly, in this work, we have only considered the bosonic part of the BornInfeld terms to the action. We neither added any higher derivative terms on the Maxwell side or considered a supersymmetric Born-Infeld lagrangian. In fact, it is not clear whether it is possible to get exact analytical results when other types of gauge-gravitational terms are included in the action. The fact that an exact calculation to all orders in $\alpha^{\prime}$ for the Born-
Infeld part is possible for two charge black holes, owes to the simplicity of the 'entropy function' formalism. It should be interesting to include other possible terms appearing in BornInfeld theories and check their dependence on entropy. All these issues remain to be pursued and we hope to return to them in future.

\section{Acknowledgements}

I wish to thank M. Alishahiha and M. M. Sheikh-Jabbari for helpful discussions and suggestions, and S. Mukherji for a useful communication. I am grateful to A. Sen for comments on the preliminary draft.
[1] D. G. Boulware and S. Deser, Phys. Rev. Lett. 55, 2656 (1985).

[2] D. L. Wiltshire, Phys. Rev. D 38, 2445 (1988).

[3] D. L. Wiltshire, Phys. Lett. B 169, 36 (1986).

[4] S. Mignemi and D. L. Wiltshire, Phys. Rev. D 46, 1475 (1992) [hep-th/9202031].

[5] M. Cvetic, S. Nojiri, and S. D. Odintsov, Nucl. Phys. B 628 295 (2002) [hep-th/0112045].

[6] Y. M. Cho and I. P. Neupane, Phys. Rev. D 66, 024044 (2002) [hep-th/0202140]; I. P. Neupane, Phys. Rev. D 67, 061501 (2003) [hep-th/0212092].

[7] V. Hubeny, A. Maloney, and M. Rangamani, [hep-th/0411272].

[8] D. Bak, S. Kim, and S. J. Rey, [hep-th/0501014].

[9] R. M. Wald, Phys. Rev. D 48, 3427 (1993) [gr-qc/9307038].

[10] T. Jacobson, G. Kang, and R. C. Myers, Phys. Rev. D 49, 6587 (1994) [gr-qc/9312023].

[11] V. Iyer and R. M. Wald, Phys. Rev. D 50, 846 (1994) [grqc/9403028]

[12] T. Jacobson, G. Kang, and R. C. Myers, [gr-qc/9502009].

[13] A. Dabholkar, R. Kallosh, and A. Maloney, JHEP 0412, 059 (2004) [hep-th/0410076].

[14] A. Sen, Mod. Phys. Lett. A 10, 2081 (1995) [hep-th/9504147].

[15] L. Susskind, L. Thorlacius, and J. Uglum, Phys. Rev. D 48, 3743 (1993) [hep-th/9306069].

[16] A. Sen, JHEP 0507, 073 (2005) [hep-th/0505122].

[17] A. Dabholkar, Phys. Rev. Lett. 94, 241301 (2005) [hepth/0409148].

[18] A. Sen, [hep-th/0411255].

[19] A. Strominger and C. Vafa, Phys. Lett. B 379, 99 (1996) [hepth/9601029].

[20] J. M. Maldacena, A. Strominger, and E. Witten, JHEP 9712, 002 (1997) [hep-th/9711053].

[21] C. Vafa, Adv. Theor. Math. Phys. 2, 207 (1998) [hepth/9711067].

[22] A. Sen, [hep-th/0502126].

[23] C. F. E. Holzhey and F. Wilczek, Nucl. Phys. B 380, 447 (1992) [hep-th/9202014].

[24] B. de Wit, Nucl. Phys. Proc. Suppl. 49, 191 (1996) [hepth/9602060].

[25] B. de Wit, Fortsch. Phys. 44, 529 (1996) [hep-th/9603191].

[26] K. Behrndt, G. L. Cardoso, and S. Mahapatra, [hepth/0506251].

[27] K. Behrndt, G. Lopes Cardoso, B. de Wit, D. Lust, T. Mo- haupt, and W. A. Sabra, Phys. Lett. B 429, 289 (1998) [hepth/9801081].

[28] G. Lopes Cardoso, B. de Wit, and T. Mohaupt, Phys. Lett. B 451, 309 (1999) [hep-th/9812082].

[29] G. Lopes Cardoso, B. de Wit, and T. Mohaupt, Fortsch. Phys. 48, 49 (2000) [hep-th/9904005].

[30] G. Lopes Cardoso, B. de Wit, and T. Mohaupt, Nucl. Phys. B 567, 87 (2000) [hep-th/9906094].

[31] G. Lopes Cardoso, B. de Wit, and T. Mohaupt, Class. Quant. Grav. 17, 1007 (2000) [hep-th/9910179].

[32] T. Mohaupt, Fortsch. Phys. 49, 3 (2001) [hep-th/0007195].

[33] G. Lopes Cardoso, B. de Wit, J. Kappeli, and T. Mohaupt, JHEP 0012, 019 (2000) [hep-th/0009234].

[34] G. L. Cardoso, B. de Wit, J. Kappeli, and T. Mohaupt, Fortsch. Phys. 49, 557 (2001) [hep-th/0012232].

[35] G. L. Cardoso, B. de Wit, J. Kappeli, and T. Mohaupt, JHEP 0412, 075 (2004) [hep-th/0412287].

[36] For a review see, A. Sen, talk in ISS2005, http://physics.ipm.ac.ir/conferences/iss2005/titles.htm.

[37] S. Ferrara, R. Kallosh, and A. Strominger, Phys. Rev. D 52, 5412 (1995) [hep-th/9508072].

[38] A. Strominger, Phys. Lett. B 383, 39 (1996) [hep-th/9602111].

[39] S. Ferrara and R. Kallosh, Phys. Rev. D 54, 1514 (1996) [hepth/9602136].

[40] S. Ferrara, G. W. Gibbons, and R. Kallosh, Nucl. Phys. B 500, 75 (1997) [hep-th/9702103].

[41] H. Ooguri, A. Strominger, and C. Vafa, Phys. Rev. D 70, 106007 (2004) [hep-th/0405146].

[42] A. Sen, [hep-th/0506177].

[43] P. Kraus and F. Larsen, JHEP 0509, 034 (2005) [hepth/0506176]; P. Kraus and F. Larsen, JHEP 0601, 022 (2006) [hep-th/0508218].

[44] K. Goldstein, N. Iizuka, R. P. Jena, and S. P. Trivedi, [hepth/0507096].

[45] P. K. Tripathy and S. P. Trivedi, [hep-th/0511117].

[46] R. Kallosh, [hep-th/0510024].

[47] K. Goldstein, R. P. Jena, G. Mandal, and S. P. Trivedi, [hepth/0512138].

[48] M. Alishahiha and H. Ebrahim, [hep-th/0601016].

[49] For a recent review, see A. Sen, Talk in Strings 2005, "http://www.fields.utoronto.ca/ audio/05-06/strings/sen/".

[50] A. Sen, [hep-th/0508042]. 
[51] B. Zwiebach, Phys. Lett. B 156, 315 (1985).

[52] J. A. Harvey and G. W. Moore, Phys. Rev. D 57, 2323 (1998) [hep-th/9610237].

[53] P. Prester, [hep-th/0511306].

[54] D. P. Jatkar and A. Sen, [hep-th/0510147].

[55] J. R. David, D. P. Jatkar, and A. Sen, [hep-th/0602254].

[56] A. Dabholkar and S. Nampuri, [hep-th/0603066].

[57] G. Lopes Cardoso, B. de Wit, J. Kappeli, and T. Mohaupt, [hepth/0601108].

[58] A. Sinha and N. V. Suryanarayana, [hep-th/0601183].

[59] B. Sahoo and A. Sen, [hep-th/0601228].

[60] R. Kallosh, N. Sivanandam, and M. Soroush, [hep-th/0602005].

[61] B. Sahoo and A. Sen, [hep-th/0603149].

[62] G. Exirifard, [hep-th/0604021].

[63] M. Born and L. Infeld, Proc. Roy. Soc. Lond. A 144, 425 (1934).

[64] E. S. Fradkin and A. A. Tseytlin, Phys. Lett. B 163, 123 (1985).

[65] A. Abouelsaood, C. G. . Callan, C. R. Nappi, and S. A. Yost, Nucl. Phys. B 280, 599 (1987).

[66] E. Bergshoeff, E. Sezgin, C. N. Pope, and P. K. Townsend, Open Superstring," Phys. Lett. 188B, 70 (1987).

[67] R. R. Metsaev, M. A. Rakhmanov, and A. A. Tseytlin, Phys. Lett. B 193, 207 (1987).

[68] A. A. Tseytlin, Phys. Lett. B 202, 81 (1988).

[69] O. D. Andreev and A. A. Tseytlin, Phys. Lett. B 207, 157 (1988).
[70] O. D. Andreev and A. A. Tseytlin, Nucl. Phys. B 311, 205 (1988).

[71] A. A. Tseytlin, Nucl. Phys. B 276 (1986) 391 [Erratum-ibid. B 291 (1987) 876].

[72] C. G. . Callan, C. Lovelace, C. R. Nappi, and S. A. Yost, Nucl. Phys. B 308, 221 (1988).

[73] A. A. Tseytlin, [hep-th/9908105].

[74] T. Tamaki and T. Torii, Phys. Rev. D 62, 061501 (2000) [grqc/0004071].

[75] M. Wirschins, A. Sood, and J. Kunz, Phys. Rev. D 63, 084002 (2001) [hep-th/0004130].

[76] G. Clement and D. Gal'tsov, Phys. Rev. D 62, 124013 (2000) [hep-th/0007228].

[77] T. Tamaki and T. Torii, Phys. Rev. D 64, 024027 (2001) [grqc/0101083].

[78] B. Chandrasekhar, S. Parvizi, A. Tavanfar, and H. Yavartanoo, [hep-th/0602022].

[79] G. W. Gibbons and D. A. Rasheed, Nucl. Phys. B 454, 185 (1995) [hep-th/9506035].

[80] D. A. Rasheed, [hep-th/9702087].

[81] M. Cvetic and D. Youm, Phys. Rev. D 53, 584 (1996) [hepth/9507090]; M. Cvetic and A. A. Tseytlin, Phys. Rev. D 53, 5619 (1996) [Erratum-ibid. D 55, 3907 (1997)] [hepth/9512031]. 